\section{Año 2019-2020. urtea N. ${ }^{\circ} 31-32 . z k$.}

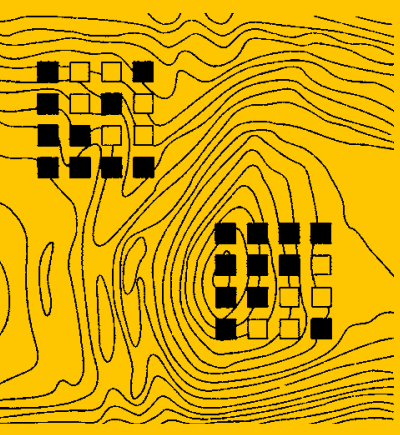

\section{TRABAJOS DE ARQUEOLOGÍA NAVARRA}

SEPARATA

\section{Arte parietal paleolítico de la cueva de Alkerdi 2} (Urdazubi/Urdax, Navarra)

D. Garate, O. Rivero, A. Hermoso de Mendoza, J. Tapia Sagarna, M. A. Medina-Alcaide, I. Álvarez, A. Aranburu, A. Bodego, M. Arriolabengoa, E. Iriarte, P. Bilbao, M. del Val, V. Abendaño, J. I. Calvo, F. Ibarra, J. Legarrea, J. Agirre-Mauleon 


\title{
Arte parietal paleolítico de la cueva de Alkerdi 2 (Urdazubi/Urdax, Navarra)
}

\author{
Alkerdi 2 kobazuloko paleolito garaiko labar artea (Urdazubi/Urdax, Navarra)
}

Palaeolithic rock art in Alkerdi 2 cave (Urdazubi/Urdax, Navarra)

D. Garate (1), O. Rivero (2), A. Hermoso de Mendoza (3), J. Tapia Sagarna (4), M. A. Medina-Alcaide (5), I. Álvarez (6, 4), A. Aranburu (6, 4), A. Bodego (6, 4), M. Arriolabengoa (6, 4), E. Iriarte (7, 4), P. Bilbao (6, 4), M. del Val $(8,4)$, V. Abendaño(3), J. I. Calvo (3), F. Ibarra (3), J. Legarrea (1), J. Agirre-Mauleon (4)

Correo de contacto: garatemaidagandiego@gmail.com

DOI: https://doi.org/10.35462/TAN31-32.25

(1) Instituto Internacional de Investigaciones Prehistóricas de Cantabria, Universidad de Cantabria. Avda. de los Castros s/n, 39005 Santander.

(2) Departamento de Prehistoria, Historia Antigua y Arqueología, Universidad de Salamanca. Cervantes s/n, 37002 Salamanca.

(3) Grupo de Espeleología Satorrak. Calle Descalzos 37 bajo bis, 31001 Iruña-Pamplona.

(4) Aranzadi Zientzia Elkartea/Sociedad de Ciencias Aranzadi. Calle Zorroagagaina, 11. 20014 Donostia-San Sebastián.

(5) Departamento de Historia, Universidad de Córdoba. 14071 Córdoba.

(6) Departamento de Geología, Facultad de Ciencia y Tecnología, Euskal Herriko Unibertsitatea/ Universidad del País Vasco. Barrio Sarriena s/n 48940 Leioa.

(7) Laboratorio de Evolución Humana, Edificio de I+D+i, Universidad de Burgos. Plaza Misael Bañuelos s/n, 09001 Burgos.

(8) Centro Nacional de Investigación sobre la Evolución Humana (CENIEH), 09002 Burgos. 


\section{RESUMEN}

En el marco del proyecto de "Caracterización del macizo y sistema kárstico de Alkerdi, de la cueva de Alkerdi y de su entorno de protección", se procede a la exploración de la cavidad de Alkerdi 2. Las labores coordinadas han permitido identificar un conjunto de arte parietal asociado a evidencias arqueológicas en superficie. El estilo de las grafías animales grabadas apunta hacia una atribución Gravetiense para la presencia humana. La datación por radiocarbono de uno de los motivos parietales y de un carbón procedente del contexto arqueológico, lo corroboran. Se trata así de la segunda cavidad decorada durante el Paleolítico en Navarra y la muestra artística más antigua de la Comunidad Foral.

Palabras clave: arte parietal; bisonte; pintura; Gravetiense; Navarra.

\section{LABURPENA}

Alkerdiko mendigune eta sistema karstikoaren zein Alkerdiko kobazuloaren eta bere babes eremuaren azterketarako proiektuaren barruan, Alkerdi 2 kobazuloaren miaketa burutu da. Ikerketa koordinatuek lurrazalean agerturiko aztarna arkeologikoekin erlazionaturiko labar arte multzo bat identifikatzea ahalbidetu dute. Grabaturiko animalien irudien estiloak Gravette aldiko giza presentzia bat frogatzen dute. Labar irudietako baten zein testuingurutik datorren ikatz baten erradiokarbonozko datazioek hau baieztatuko lukete. Beraz, Nafarroan agerturiko bigarren kobazulo apainduaren aurrean egongo ginateke, eta Foru Erkidegoko adibide artistiko zaharrenen aurrean.

Gako hitzak: Labar artea; bisonte; margoa; Gravetiar aldia; Nafarroa.

\section{ABSTRACT}

Within the framework of the characterization project of the massif and karst system of Alkerdi, the Alkerdi cave and its protective environment, the Alkerdi 2 cavity was explored. The coordinated work has made it possible to identify a set of parietal art associated with archaeological evidence on the surface. The style of the engraved animal points towards a Gravettian attribution for human presence. Radiocarbon dating of one of the parietal motifs and a charcoal from the archaeological context corroborate this idea. This is the second cavity decorated during the Paleolithic in Navarre and the oldest artistic remain in the Autonomous Community.

Keywords: Rock art; bison; paintings; Gravettian; Navarre. 
El macizo kárstico de Alkerdi está localizado al norte de la Comunidad Foral de Navarra, en el municipio de Urdazubi/Urdax, sobre una unidad calcárea de la base del Cretácico Superior (de edad Albiense a Turoniense), con una orientación cartográfica general SO-NE. Se abre hacia la vertiente norte del extremo occidental de los Pirineos y su altitud apenas supera los $100 \mathrm{~m}$.

Las investigaciones arqueológicas en el macizo se habían centrado en los yacimientos de ocupación humana del abrigo de Berroberria y la cueva vecina de Alkerdi 1 (Barandiaran et al., 2010). Además, en la segunda, se conocía un conjunto de arte parietal paleolítico (Casteret, 1933; Barandiaran, 1974), que recientemente ha sido revisado y ampliado de manera significativa (Garate \& Rivero, 2014).

La Dirección General de Cultura-Institución Príncipe de Viana del Gobierno de Navarra impulso y promovió el proyecto denominado «Caracterización del macizo y sistema kárstico de Alkerdi, de la cueva de Alkerdi y de su entorno de protección» que fue desarrollado entre los años 2015-2017. Gracias a sus importantes resultados durante los años 2018-2020 el Ayuntamiento de Urdazubi-Urdax, con el apoyo del Gobierno de Navarra, ha continuado promoviendo estas investigaciones dirigidas por la Sociedad de Ciencias Aranzadi. En este contexto de investigación y de políticas institucionales se ha procedido a la exploración arqueológica de la cavidad de Alkerdi 2, cuyo acceso permanecía protegido mediante un enrejado.

El 28 de marzo de 2016 se localizan de una serie de motivos en negro sobre el techo de una de las salas de la cueva de Alkerdi 2, por parte de Jesús Tapia (Aranzadi Zientzia Elkartea) e inmediatamente después el signo triangular por Arturo Hermoso de Mendoza 
(Satorrak Espeleologia Elkartea), y tres días después una serie de puntos rojos en otra galería lateral por Jaime Legarrea (Satorrak Espeleologia Elkartea). Al mismo tiempo, se observa la presencia de herramientas de sílex, huesos y restos carbonosos en diversos puntos de la cavidad. En visita el día 1 de abril se realiza una primera evaluación de los motivos por parte del especialista Diego Garate, confirmando su atribución prehistórica.

De esta manera, Alkerdi 2 se convierte en la segunda cavidad navarra con arte parietal paleolítico, después del descubrimiento de Alkerdi 1 en 1930. Tras una primera evaluación preliminar en 2016, se desarrollan varias campañas de trabajo de campo entre 2018 y 2020, con la finalidad de prospectar y documentar las evidencias arqueológicas de toda la cavidad. El 6 de julio de 2020 durante la prospección espeleológica de la cavidad se localiza un nuevo sector decorado por parte de Arturo Hermoso de Mendoza e Israel Robles (Satorrak Espeleologia Elkartea).

La cavidad cuenta con un impacto humano casi nulo, sin ningún tipo de material antrópico ni restos de pisadas, roces u otras marcas de progresión, a excepto de las prehistóricas (sílex, huesos, carbones) y de las dejadas por el equipo de trabajo durante las jornadas de exploración. Solamente en el nivel freático, por donde discurre el río subterráneo, se detectan algunos materiales actuales. Es decir, en los pisos superiores se trata de una cueva virgen, con las implicaciones que eso conlleva de cara al estudio de un registro arqueológico in situ. Por motivos de conservación y para no alterar el estado actual de la cavidad, hemos limitado nuestras visitas a las vías ya transitadas por el equipo, debidamente balizadas y señalizadas, sin salirse de las mismas para evitar generar nuevas huellas o destruir evidencias arqueológicas poco visibles.

Las evidencias de arte parietal paleolítico de la cueva de Alkerdi 2 se localizan en cuatro sectores diferentes de su recorrido. Dos de ellos -A y B- concentran la mayoría de las representaciones y se localizan contiguos, al fondo de la cavidad con respecto a la entrada actual. Los otros dos sectores se localizan en sendos desarrollados laterales, alejados del área de tránsito principal de la cavidad. A ello se suma un quinto sector localizado en 2020 y todavía pendiente de estudio.

En total se trata de 21 entidades gráficas de las que 3 son representaciones animales grabadas (bisonte, cuadrúpedo indeterminado y caballo), 3 signos complejos (parrilla en rojo, y triángulo y nube de puntos en negro) y el resto líneas grabadas, y puntos y manchas en rojo. A todo ello debemos añadir el nuevo sector decorado localizado en 2020, todavía en proceso de estudio y que, presumiblemente, doble el número total de evidencias gráficas parietales de la cavidad. En una valoración preliminar y, por lo tanto incompleta, hemos identificado diez paneles decorados con una cantidad variable de figuras en cada uno de ellos -entre 1 y 6 -. Destaca de manera notable un panel compuesto por representaciones de vulvas, un panel que contiene cinco bisontes grabados, y otro panel con dos caballos. En todos los casos se ha recurrido al grabado, excepto para una serie de tres trazos pareados de color rojo.

Además, en el interior de la cavidad se han registrado materiales arqueológicos sobre la superficie en dos áreas: una primera zona en el cono de derrubios inmediato a 
la entrada con fauna, carbones y alguna pieza lítica, y una segunda zona en un piso inferior intermedio donde se ubican la mayoría de las evidencias, en el sector decorado B. En él se han identificado concentraciones de carbón, un hogar compuesto por hueso quemado y restos de talla, y media docena de piezas de sílex dispersas.

El estilo del arte parietal y en concreto de los bisontes grabados, coincide con el registro conocido para época gravetiense (circa 28 000-20 000 años BP). En concreto, la manera de representar la cornamenta y los detalles anatómicos, son coumunes para una serie de cavidades del Suroeste francés y con especial incidencia en los Pirineos, incluyendo las cuevas de la colina de Aitzbitarte en Gipuzkoa (Garate et al., 2020).

Con el fin de aquilatar la atribución cronológica de las actividades humanas desarrolladas en la cueva de Alkerdi 2, se ha datado una muestra de carbón de la figura ALK2.B.II.4 y de un resto carbonoso situado a pie de panel. Si bien la primera contaba con escasa materia datable, nos arroja una fecha ante quem que es totalmente compatible con el resultado de la segunda, fechada en torno a 27000 años. En todo caso, se han enviado nuevas muestras al laboratorio de Oxford Radiocarbon Accelerator Unit (Reino Unido), con la finalidad de corroborar los resultados. Caber recordar que la propia cueva de Alkerdi 1 presenta ocupaciones humanas de dicha cronología con una fechación en torno a 26000 BP (Barandiarán et al., 2010).

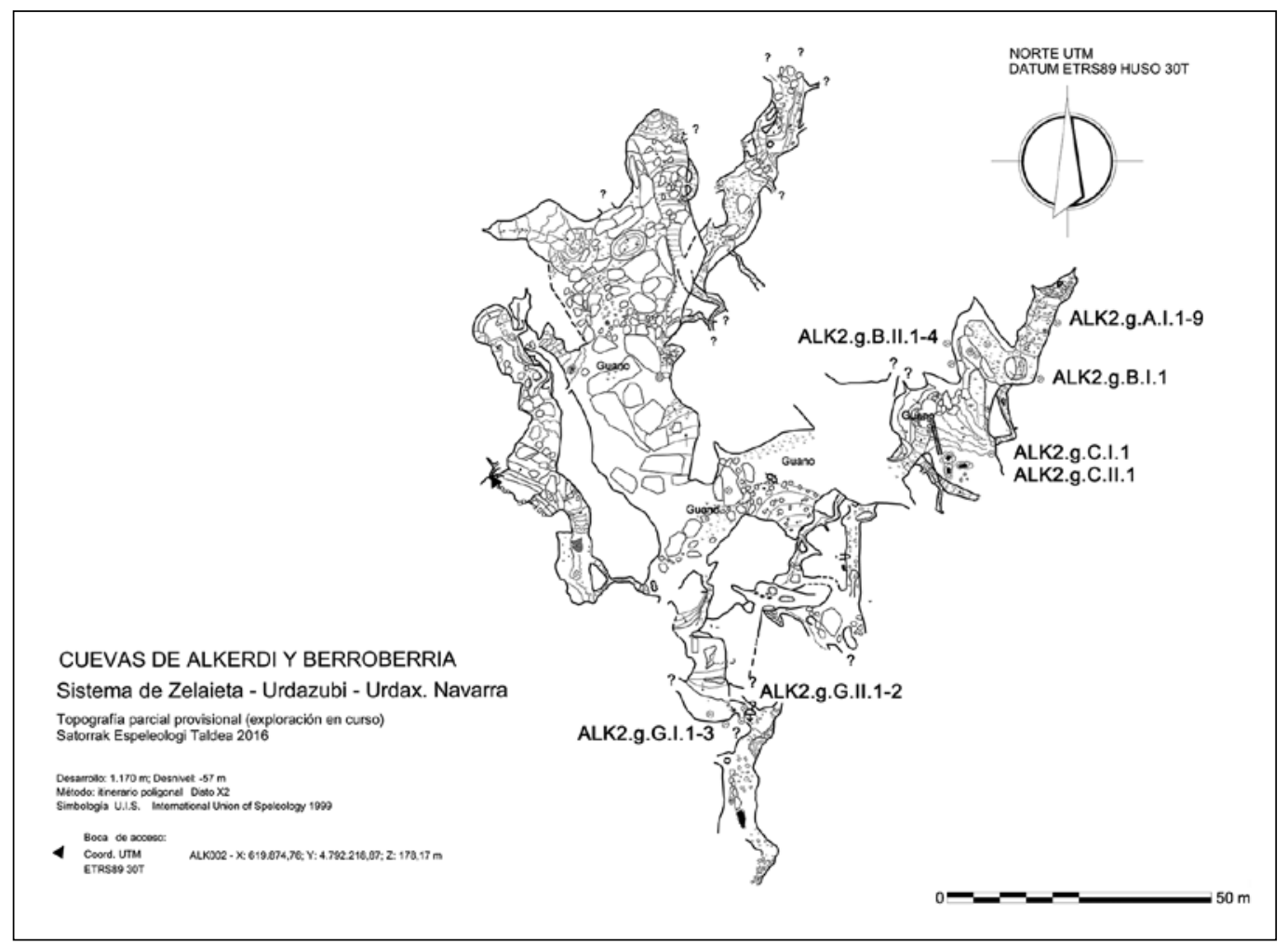

Figura 1. Localización de las unidades gráficas documentadas sobre el plano de la cavidad. Satorrak Espeleologia Taldea ${ }^{\odot}$. 
En definitiva, Alkerdi 2 es la segunda cueva con arte parietal paleolítico descubierta en Navarra -la primera fue Alkerdi 1 en 1930- e, indudablemente ante la muestra más antigua de arte de toda la Comunidad Foral. Si bien Alkerdi 1 presenta un importante conjunto de grabados magdalenienses, la mayoría descubiertos recientemente (Garate \& Rivero, 2014), los nuevos descubrimientos producidos en Alkerdi 2 durante 2020, otorgan a ambas cavidades la misma relevancia en cuanto a la amplitud de su catálogo gráfico y a su aportación para el estudio del arte paleolítico europeo. Es decir, ambas cavidades presentan un interés científico muy relevante.

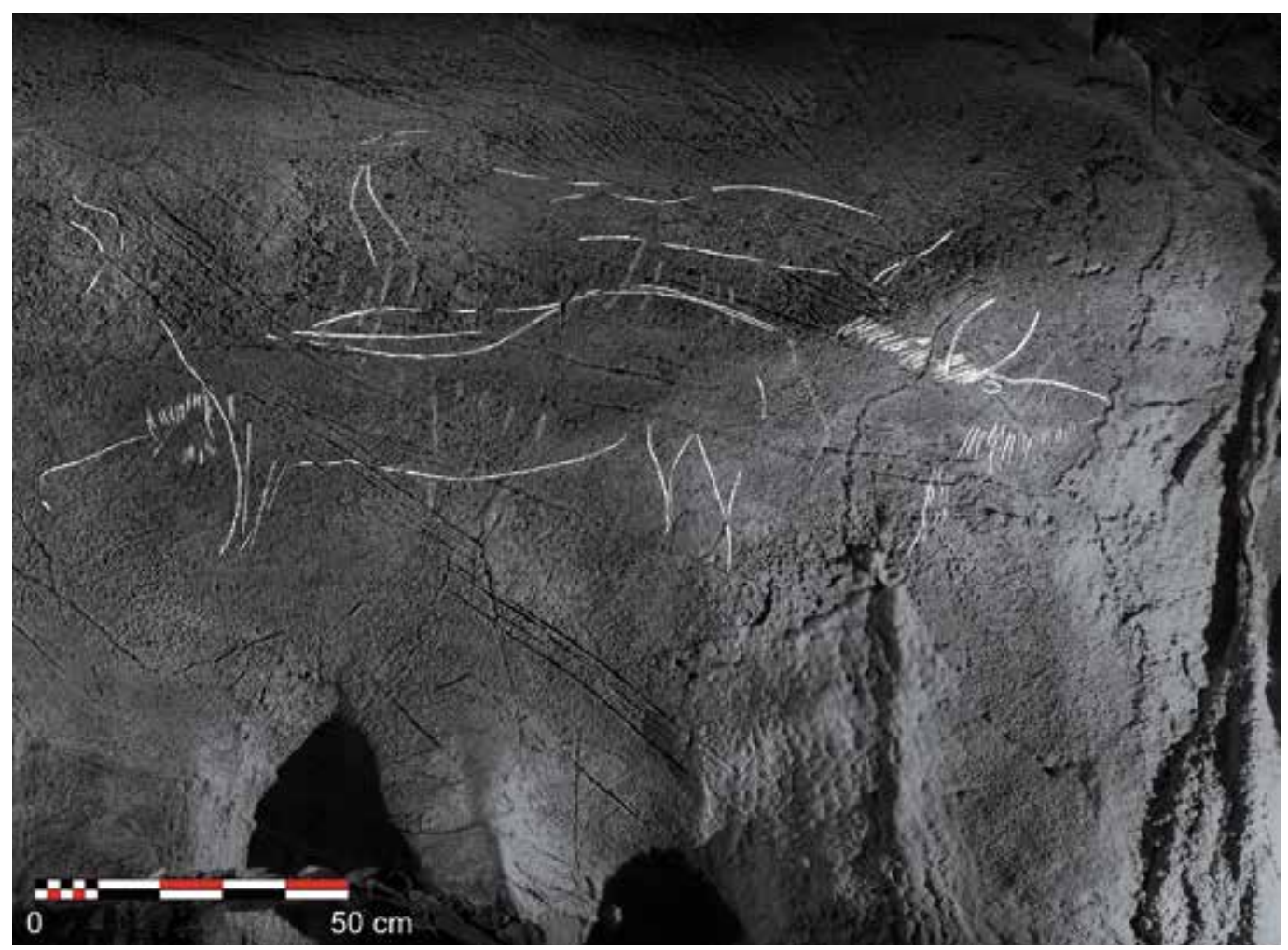

Figura 2. Calco del bisonte (ALK2.g.A.I.1), animal indeterminado (ALK2.g.A.I.2) y líneas curvas grabadas (ALK2.g.A.I.3) en la pared derecha del sector A.

\section{REFERENCIAS BIBLIOGRÁFICAS}

Alvarez, I., Abendaño, V., Aranburu, A., Arriolabengoa, M., Bodego, A., Calvo, J. I., Garate Maidagan, D., García-García, E., Hermoso de Mendoza, A., Ibarra, F., Iriarte, E., Legarrea, J., Tapia, J., del Val, M. \& Agirre, J. (2016). Estudio interdisciplinar del macizo kárstico de Alkerdi: rasgos geológicos, evolución kárstica y contenido arqueopaleontológico. Trabajos de Arqueología de Navarra, 28, 273-307.

Barandiarán, I. (1974). Arte paleolítico en Navarra. Las cuevas de Urdax. Príncipe de Viana, 134-135, 9-47. 
Barandiarán, I., Cava, A. \& Elorrieta, I. (2010). Alternancia/complementariedad en las cuevas de Alkerdi y Berroberría. Cuadernos de Navarra, 18, 9-40.

Casteret, N. (1933). Une nouvelle grotte à gravures dans les Pyrénées. La grotte d'Alquerdi. En XVe Congrès International d'Anthropologie et d'Archéologie Préhistorique. Paris: 20-27 septembre 1931 (pp. 384-389). París: Librairie E. Nourry.

Garate, D. (2017). New insights into the study of Palaeolithic rock art: Dismantling the 'Basque Country Void'. Journal of Anthropological Research, 74(2), 168-200.

Garate, D. \& Rivero, O. (2014). La galería de los bisontes: un nuevo sector decorado en la cueva de Alkerdi (Urdazubi/Urdax, Navarra). Zephyrus, 75, 17-39.

Garate, D., Rivero, O., Ruiz-Redondo, A. \& Rios-Garaizar, J. (2014). At the crossroads: A new approach to the Upper Paleolithic art in the Western Pyrenees. Quaternary International, 364, 283-293.

Garate, D., Tapia, J., Rivero, O., Álvarez, I., Abendaño, V., Aranburu, A., Arriolabengoa, M., Bodego, A., Calvo, J. I., Garcia-Garcia, E., Hermoso de Mendoza, A., Ibarra, F., Iriarte, E., Legarrea, J., Del Val, M. \& Agirre, J. (2017). Alkerdi 2: une grotte ornée gravettien dans les Pyrenées occidentales. International Newsletter on Rock Art, 79, 10-12.

Garate, D., Rivero, O., Rios-Garaizar, J., Arriolabengoa, M., Intxaurbe, I. \& Salazar, S. (2020). Redefining shared symbolic networks during the Gravettian in Western Europe: new data from the rock art findings in Aitzbitarte caves (NorthernSpain). PLoS ONE, 15(10): e0240481. https://doi.org/10.1371/ journal.pone.0240481 